Research Article

\title{
Impulsive Boundary Value Problems for Planar Hamiltonian Systems
}

\author{
Zeynep Kayar and Ağacık Zafer \\ Department of Mathematics, Middle East Technical University, 06800 Ankara, Turkey \\ Correspondence should be addressed to Zeynep Kayar; zkayar@metu.edu.tr \\ Received 3 September 2013; Accepted 24 October 2013 \\ Academic Editor: Josef Diblik
}

Copyright (c) 2013 Z. Kayar and A. Zafer. This is an open access article distributed under the Creative Commons Attribution License, which permits unrestricted use, distribution, and reproduction in any medium, provided the original work is properly cited.

We give an existence and uniqueness theorem for solutions of inhomogeneous impulsive boundary value problem (BVP) for planar Hamiltonian systems. Green's function that is needed for representing the solutions is obtained and its properties are listed. The uniqueness of solutions is connected to a Lyapunov type inequality for the corresponding homogeneous BVP.

\section{Introduction}

The planar Hamiltonian system of 2-linear first-order equations has the form

$$
y^{\prime}=J H(t) y, \quad t \in \mathbb{R},
$$

where

$$
H(t)=\left[\begin{array}{ll}
c(t) & a(t) \\
a(t) & b(t)
\end{array}\right]
$$

is a symmetric matrix with piecewise continuous real-valued entries, and

$$
J=\left[\begin{array}{cc}
0 & 1 \\
-1 & 0
\end{array}\right]
$$

is the so called symplectic identity. Setting

$$
y_{1}(t)=x(t), \quad y_{2}(t)=u(t),
$$

the Hamiltonian system can be rewritten in a more convenient way as

$$
\begin{aligned}
& x^{\prime}=a(t) x+b(t) u, \\
& u^{\prime}=-c(t) x-a(t) u .
\end{aligned}
$$

Our aim in this work is to prove an existence and uniqueness theorem for solutions of the related BVP for inhomogeneous Hamiltonian system under impulse effect of the form

$$
\begin{array}{r}
x^{\prime}=a(t) x+b(t) u+f_{1}(t), \\
u^{\prime}=-c(t) x-a(t) u+f_{2}(t), \\
t \in\left(t_{1}, t_{2}\right) \backslash\left\{\tau_{i}\right\},
\end{array}
$$

$$
\begin{gathered}
x\left(\tau_{i}^{+}\right)=\alpha_{i} x\left(\tau_{i}^{-}\right)+a_{i 1}, \\
u\left(\tau_{i}^{+}\right)=-\beta_{i} x\left(\tau_{i}^{-}\right)+\alpha_{i} u\left(\tau_{i}^{-}\right)+a_{i 2}, \\
i=1,2, \ldots, p, \\
x\left(t_{1}\right)=A, \quad x\left(t_{2}\right)=B,
\end{gathered}
$$

where

(i) $\left\{\tau_{i}\right\},\left\{\alpha_{i}\right\},\left\{\beta_{i}\right\},\left\{a_{i 1}\right\}$, and $\left\{a_{i 2}\right\}$ are real sequences for $i=1,2, \ldots, p$ with

$$
t_{1}<\tau_{1}<\tau_{2}<\cdots<\tau_{p}<t_{2} ;
$$

(ii) $a, b, c, f_{1}, f_{2} \in \operatorname{PLC}\left(J_{0}\right)$, where $J_{0}=\left[t_{1}, t_{2}\right]$ and $\operatorname{PLC}\left(J_{0}\right)=\left\{\omega: J_{0} \rightarrow \mathbb{R}\right.$ is continuous on each interval $\left(\tau_{i}, \tau_{i+1}\right)$, the limits $w\left(\tau_{i}^{ \pm}\right)$exist and $w\left(\tau_{i}^{-}\right)=$ $w\left(\tau_{i}\right)$ for $\left.i=1,2, \ldots, p\right\}$;

(iii) $b(t)>0$ for $t \in\left(t_{1}, t_{2}\right)$ and $\alpha_{i} \neq 0$ for $i=1,2, \ldots$, $p ; A$ and $B$ are given real numbers.

We also set $\tau_{0}=t_{1}$ and $\tau_{p+1}=t_{2}$ for convenience. 
By a solution of the impulsive BVP $(6 a)-(6 c)$, we mean nontrivial functions $x, u \in \operatorname{PLC}\left(J_{0}\right)$ such that $(x, u)$ satisfies system (6a)-(6c) for all $t \in J_{0}$.

The corresponding homogeneous BVP takes the form

$$
\begin{gathered}
x^{\prime}=a(t) x+b(t) u, \quad u^{\prime}=-c(t) x-a(t) u, \\
t \in\left(t_{1}, t_{2}\right) \backslash\left\{\tau_{i}\right\}, \\
x\left(\tau_{i}^{+}\right)=\alpha_{i} x\left(\tau_{i}^{-}\right), \quad u\left(\tau_{i}^{+}\right)=-\beta_{i} x\left(\tau_{i}^{-}\right)+\alpha_{i} u\left(\tau_{i}^{-}\right), \\
i=1,2, \ldots, p, \\
x\left(t_{1}\right)=0, \quad x\left(t_{2}\right)=0 .
\end{gathered}
$$

Note that if we take

$$
\begin{gathered}
a(t) \equiv 0, \quad b(t)=\frac{1}{p(t)}, \quad c(t)=q(t), \\
f_{1}(t) \equiv 0, \quad f_{2}(t)=f(t),
\end{gathered}
$$

then we obtain as a special case of (6a), (6b), and (6c) the impulsive BVP for second-order differential equations of the form

$$
\begin{aligned}
&\left(p(t) x^{\prime}\right)^{\prime}+q(t) x=f(t), \quad t \in\left(t_{1}, t_{2}\right) \backslash\left\{\tau_{i}\right\}, \\
& x\left(\tau_{i}^{+}\right)=\alpha_{i} x\left(\tau_{i}^{-}\right)+a_{i 1}, \\
&\left(p x^{\prime}\right)\left(\tau_{i}^{+}\right)=-\beta_{i} x\left(\tau_{i}^{-}\right)+\alpha_{i}\left(p x^{\prime}\right)\left(\tau_{i}^{-}\right) \\
&+a_{i 2}, \quad i=1,2, \ldots, p, \\
& x\left(t_{1}\right)=A, \quad x\left(t_{2}\right)=B .
\end{aligned}
$$

To the best of our knowledge although many results have been obtained for linear impulsive boundary value problems by using different techniques, there is little known for the linear $2 \times 2$ Hamiltonian systems under impulse effect.

The existence and uniqueness of linear impulsive boundary value problem for the first-order equations are considered in [1-4]. For the second-order case we refer to $[5,6]$ in which the integral representation of the solution of second order linear impulsive boundary value problems is given by using Green's function and the existence and uniqueness of the solutions are obtained. Variational technique approach for the existence of the solutions of linear and nonlinear impulsive boundary value problems can be found in [7-10]. In [11], the method of upper and lower solutions is employed for the existence of solutions of nonlinear impulsive boundary value problems. For a detailed discussion on boundary value problems for higher-order linear impulsive equations we refer to [12]. Basic theory of impulsive differential equations is contained in the seminal book [13].

Our method of proof is based on Green's function formulation and Lyapunov type inequalities for linear Hamiltonian system under impulse effect. There are many studies on Lyapunov type inequalities and their applications for linear ordinary differential equations [14] and for systems [15-17] as well as for linear impulsive differential equations and systems $[18,19]$. However, the use of a Lyapunov type inequality in connection with BVPs seems to be limited.

\section{Preliminaries}

2.1. Lyapunov Type Inequality for Homogeneous Problem. In this section we provide a Lyapunov type inequality to be used for the uniqueness of the inhomogeneous BVP. The obtained inequality is sharper than the one given by the present authors in [20] in the sense that $2|a(t)|$ is replaced by $|a(t)|$.

Theorem 1. If the homogeneous BVP (8a), (8b), and (8c) has a real solution $(x(t), u(t))$ such that $x(t) \neq 0$ on $\left(t_{1}, t_{2}\right)$, then one has the Lyapunov type inequality:

$$
\begin{aligned}
& \exp \left(\int_{t_{1}}^{t_{2}}|a(t)| d t\right)\left[\int_{t_{1}}^{t_{2}} b(t) d t\right] \\
& \quad \times\left[\int_{t_{1}}^{t_{2}} c^{+}(t) d t+\sum_{t_{1} \leq \tau_{i}<t_{2}}\left(\frac{\beta_{i}}{\alpha_{i}}\right)^{+}\right] \geq 4,
\end{aligned}
$$

where $c^{+}(t)=\max \{c(t), 0\}$ and $\left(\beta_{i} / \alpha_{i}\right)^{+}=\max \left\{\beta_{i} / \alpha_{i}, 0\right\}$.

Proof. Define

$$
z(t)=\frac{1}{\alpha_{1} \alpha_{2} \cdots \alpha_{i}} x(t), \quad v(t)=\frac{1}{\alpha_{1} \alpha_{2} \cdots \alpha_{i}} u(t)
$$

for $t \in\left(\tau_{i}, \tau_{i+1}\right)$ and $i=0,1, \ldots, p$, where we put again $\tau_{0}=$ $t_{1}, \tau_{p+1}=t_{2}$ and make a convention that $\alpha_{1} \alpha_{2} \cdots \alpha_{i}=$ 1 if $i=0$.

It is not difficult to see from (8a), (8b), (8c), and (12) that

$$
\begin{gathered}
z^{\prime}=a(t) z+b(t) v, \quad v^{\prime}=-c(t) z-a(t) v, \\
t \in\left(t_{1}, t_{2}\right) \backslash\left\{\tau_{i}\right\}, \\
z\left(\tau_{i}^{+}\right)=z\left(\tau_{i}^{-}\right), \\
v\left(\tau_{i}^{+}\right)=-\frac{\beta_{i}}{\alpha_{i}} z\left(\tau_{i}^{-}\right)+v\left(\tau_{i}^{-}\right), \\
\quad i=1,2, \ldots, p, \\
z\left(t_{1}\right)=0, \quad z\left(t_{2}\right)=0 .
\end{gathered}
$$

Since we assumed that $z\left(\tau_{i}\right)=z\left(\tau_{i}^{-}\right), z(t)$ is continuous on $\left[t_{1}, t_{2}\right]$. Moreover, $z^{\prime} \in \operatorname{PLC}\left(J_{0}\right), z\left(t_{1}\right)=z\left(t_{2}\right)=0$, and $z(t) \neq \equiv$ for all $t \in\left(t_{1}, t_{2}\right)$. We may assume without loss of generality that $z(t) \geq 0$ on $\left(t_{1}, t_{2}\right)$.

Using (13) and (14) we obtain

$$
\begin{aligned}
& (v z)^{\prime}=-c(t) z^{2}+b(t) v^{2}, \quad t \neq \tau_{i}, \\
& (v z)\left(\tau_{i}^{+}\right)-(v z)\left(\tau_{i}^{-}\right)=-\frac{\beta_{i}}{\alpha_{i}} z^{2}\left(\tau_{i}\right) .
\end{aligned}
$$

Integrating (16) from $t_{1}$ to $t_{2}$ and using (15) and (17) lead to

$$
\sum_{t_{1} \leq \tau_{i}<t_{2}} \frac{\beta_{i}}{\alpha_{i}} z^{2}\left(\tau_{i}\right)=\int_{t_{1}}^{t_{2}}\left[b(t) v^{2}(t)-c(t) z^{2}(t)\right] \mathrm{d} t,
$$


from which we have

$$
\begin{aligned}
\int_{t_{1}}^{t_{2}} b(t) v^{2}(t) \mathrm{d} t \leq & \int_{t_{1}}^{t_{2}} c^{+}(t) z^{2}(t) \mathrm{d} t \\
& +\sum_{t_{1} \leq \tau_{i}<t_{2}}\left(\frac{\beta_{i}}{\alpha_{i}}\right)^{+} z^{2}\left(\tau_{i}\right) .
\end{aligned}
$$

On the other hand, from the first equation in (13), we have

$$
\begin{gathered}
{\left[z(t) \exp \left(-\int_{t_{1}}^{t} a(u) \mathrm{d} u\right)\right]^{\prime}=b(t) v(t) \exp \left(-\int_{t_{1}}^{t} a(u) \mathrm{d} u\right),} \\
{\left[z(t) \exp \left(\int_{t}^{t_{2}} a(u) \mathrm{d} u\right)\right]^{\prime}=b(t) v(t) \exp \left(\int_{t}^{t_{2}} a(u) \mathrm{d} u\right) .}
\end{gathered}
$$

Let

$$
\max \left\{|z(t)|: t \in\left(t_{1}, t_{2}\right)\right\}=z\left(t_{0}\right)>0 .
$$

If we integrate (20) from $t_{1}$ to $t_{0}$, we see that

$$
z\left(t_{0}\right)=\int_{t_{1}}^{t_{0}} b(t) v(t) \exp \left(\int_{t}^{t_{0}} a(u) \mathrm{d} u\right) \mathrm{d} t
$$

and so

$$
z\left(t_{0}\right) \leq \int_{t_{1}}^{t_{0}} b(t)|v(t)| \exp \left(\int_{t}^{t_{0}}|a(u)| \mathrm{d} u\right) \mathrm{d} t .
$$

Using the obvious estimate

$$
\int_{t}^{t_{0}}|a(u)| \mathrm{d} u \leq \int_{t_{1}}^{t_{0}}|a(u)| \mathrm{d} u
$$

and then applying Cauchy-Schwarz inequality, we have

$$
\begin{aligned}
z^{2}\left(t_{0}\right) \leq & \exp \left(2 \int_{t_{1}}^{t_{0}}|a(u)| \mathrm{d} u\right)\left[\int_{t_{1}}^{t_{0}} b(t) \mathrm{d} t\right] \\
& \times\left[\int_{t_{1}}^{t_{0}} b(t) v^{2}(t) \mathrm{d} t\right] .
\end{aligned}
$$

Similarly, by integrating (21) from $t_{0}$ to $t_{2}$ and following the above procedure, we get

$$
\begin{aligned}
z^{2}\left(t_{0}\right) \leq & \exp \left(2 \int_{t_{0}}^{t_{2}}|a(u)| \mathrm{d} u\right)\left[\int_{t_{0}}^{t_{2}} b(t) \mathrm{d} t\right] \\
& \times\left[\int_{t_{0}}^{t_{2}} b(t) v^{2}(t) \mathrm{d} t\right] .
\end{aligned}
$$

Now we recall the elementary inequality:

$$
\frac{x^{2}}{\alpha}+\frac{y^{2}}{\beta} \geq 4 x y, \quad \alpha, \beta>0, \alpha+\beta=1
$$

for $x \geq 0$ and $y \geq 0$. In view of (26) and (27) setting

$$
\begin{gathered}
\alpha=\frac{\int_{t_{1}}^{t_{0}} b(t) \mathrm{d} t}{\int_{t_{1}}^{t_{2}} b(t) \mathrm{d} t}, \quad \beta=\frac{\int_{t_{0}}^{t_{2}} b(t) \mathrm{d} t}{\int_{t_{1}}^{t_{2}} b(t) \mathrm{d} t}, \\
x=z\left(t_{0}\right) \exp \left(-\int_{t_{1}}^{t_{0}}|a(u)| \mathrm{d} u\right), \\
y=z\left(t_{0}\right) \exp \left(-\int_{t_{0}}^{t_{2}}|a(u)| \mathrm{d} u\right)
\end{gathered}
$$

we have

$$
\frac{4 z^{2}\left(t_{0}\right)}{\exp \left(\int_{t_{1}}^{t_{2}}|a(t)| \mathrm{d} t\right)} \leq\left[\int_{t_{1}}^{t_{2}} b(t) \mathrm{d} t\right]\left[\int_{t_{1}}^{t_{2}} b(t) v^{2}(t) \mathrm{d} t\right] .
$$

Combining (19) and (30) results in

$$
\begin{aligned}
& \frac{4 z^{2}\left(t_{0}\right)}{\exp \left(\int_{t_{1}}^{t_{2}}|a(t)| \mathrm{d} t\right)} \\
& \leq\left[\int_{t_{1}}^{t_{2}} b(t) \mathrm{d} t\right] \\
& \quad \times\left[\int_{t_{1}}^{t_{2}} c(t) z^{2}(t) \mathrm{d} t+\sum_{t_{1} \leq \tau_{i}<t_{2}}\left(\frac{\beta_{i}}{\alpha_{i}}\right) z^{2}\left(\tau_{i}\right)\right] .
\end{aligned}
$$

Finally, since $z\left(t_{0}\right) \geq z(t)$ for $t \in\left[t_{1}, t_{2}\right]$, from (31) we obtain the desired inequality:

$$
\begin{aligned}
\exp & \left(\int_{t_{1}}^{t_{2}}|a(t)| \mathrm{d} t\right)\left[\int_{t_{1}}^{t_{2}} b(t) \mathrm{d} t\right] \\
\times & \times\left[\int_{t_{1}}^{t_{2}} c^{+}(t) \mathrm{d} t+\sum_{t_{1} \leq \tau_{i}<t_{2}}\left(\frac{\beta_{i}}{\alpha_{i}}\right)^{+}\right] \geq 4 .
\end{aligned}
$$

2.2. Green's Function. Here we derive Green's function to be used for the representation of the solutions of the inhomogeneous BVP.

Let

$$
\Phi(t)=\left[\begin{array}{ll}
x_{1}(t) & x_{2}(t) \\
u_{1}(t) & u_{2}(t)
\end{array}\right], \quad \Phi(0)=I
$$

be a fundamental matrix for (8a), (8b) and set

$$
M=\left[\begin{array}{ll}
1 & 0 \\
0 & 0
\end{array}\right], \quad N=\left[\begin{array}{ll}
0 & 0 \\
1 & 0
\end{array}\right] .
$$

Define the rectangles

$$
\begin{aligned}
& R_{11}=\left[t_{1}, \tau_{1}\right] \times\left[t_{1}, \tau_{1}\right], \\
& R_{i 1}=\left(\tau_{i-1}, \tau_{i}\right] \times\left[t_{1}, \tau_{1}\right], \quad i=2,3, \ldots, p+1, \\
& R_{1 j}=\left[t_{1}, \tau_{1}\right] \times\left(\tau_{j-1}, \tau_{j}\right], \quad j=2,3, \ldots, p+1, \\
& R_{i j}=\left(\tau_{i-1}, \tau_{i}\right] \times\left(\tau_{j-1}, \tau_{j}\right], \quad i, j=2,3, \ldots, p+1,
\end{aligned}
$$


and the triangles

$$
\begin{aligned}
T^{u} & =\left\{(t, s) \in\left[t_{1}, t_{2}\right] \times\left[t_{1}, t_{2}\right]: s>t\right\}, \\
T^{l} & =\left\{(t, s) \in\left[t_{1}, t_{2}\right] \times\left[t_{1}, t_{2}\right]: s<t\right\}, \\
T_{i i}^{u} & =\left\{(t, s) \in R_{i i}: s>t\right\}, \quad i=1,2,3, \ldots, p+1, \\
T_{i i}^{l} & =\left\{(t, s) \in R_{i i}: s<t\right\}, \quad i=1,2,3, \ldots, p+1 .
\end{aligned}
$$

Green's function (pair) and its properties are given in the next theorem.

Theorem 2. Suppose that the homogeneous BVP (8a)-(8c) has only the trivial solution. Let

$$
K=-\left[M \Phi\left(t_{1}\right)+N \Phi\left(t_{2}\right)\right]^{-1} N \Phi\left(t_{2}\right) .
$$

Note that the inverse of matrix $M \Phi\left(t_{1}\right)+N \Phi\left(t_{2}\right)$ exists in view of the assumption (see also the proof of Theorem 4).

Then the pair of functions

$$
\begin{gathered}
G(t, s)= \begin{cases}\Phi(t)(I+K) \Phi^{-1}(s), & s<t, \\
\Phi(t) K \Phi^{-1}(s), & s \geq t,\end{cases} \\
\widetilde{G}\left(t, \tau_{i}^{+}\right)= \begin{cases}\Phi(t)(I+K) \Phi^{-1}\left(\tau_{i}^{+}\right), & \tau_{i}<t, \\
\Phi(t) K \Phi^{-1}\left(\tau_{i}^{+}\right), & \tau_{i} \geq t,\end{cases}
\end{gathered}
$$

constitutes Green's function for (6a), (6b), and (6c). Moreover, we have the following properties:

(G1) $G(t, s)$ is continuous and bounded on $R_{i j}$,

(G2) $(\partial G(t, s)) / \partial t$ is continuous and bounded on the rectangles $R_{i j}$ with $i \neq j$ and on the triangles $T_{i i}^{u}$ and $T_{i i}^{l}$,

(G3) $G(t, s)$ satisfies the following jump conditions:

(a) $G\left(\tau_{i}^{+}, \tau_{i}\right)-G\left(\tau_{i}^{-}, \tau_{i}\right)=B_{i}+\left(B_{i}-I\right) G\left(\tau_{i}^{-}, \tau_{i}\right)$ where $B_{i}=\left[\begin{array}{cc}\alpha_{i} & 0 \\ -\beta_{i} & \alpha_{i}\end{array}\right]$,

(b) $G\left(s^{+}, s\right)-G\left(s^{-}, s\right)=I, s \neq \tau_{i}$,

(c) $\left(\partial G\left(s^{+}, s\right) / \partial t\right)-\left(\partial G\left(s^{-}, s\right) / \partial t\right)=J H(s), s \neq \tau_{i}$,

(G4) $G(t, s)$, considered as a function of $t$, is left continuous and satisfies

$$
\begin{gathered}
y^{\prime}=J H(t) y, \quad t \in J_{s} \backslash\left\{\tau_{i}\right\}, \\
y\left(\tau_{i}^{+}\right)=B_{i} y\left(\tau_{i}^{-}\right), \quad i \in\left\{i: \tau_{i} \in J_{s}\right\}, \\
M y\left(t_{1}\right)+N y\left(t_{2}\right)=0,
\end{gathered}
$$

where $J_{s}$ is any of the intervals $\left[t_{1}, s\right)$ or $\left(s, t_{2}\right]$,

(G5) $\left.\Delta\right|_{t=\tau_{i}} \widetilde{G}\left(t, \tau_{i}^{+}\right)=\widetilde{G}\left(\tau_{i}^{+}, \tau_{i}^{+}\right)-\widetilde{G}\left(\tau_{i}^{-}, \tau_{i}^{+}\right)=\left(B_{i}-\right.$ I) $\widetilde{G}\left(\tau_{i}^{-}, \tau_{i}^{+}\right)$,

(G6) $\widetilde{G}(t, s)$, considered as a function of $t$, is left continuous and satisfies (39).
Proof. (G1) and (G2) are trivial. Let us consider (G3)(a) follows from

$$
\begin{aligned}
& G\left(\tau_{i}^{+}, \tau_{i}\right)-G\left(\tau_{i}^{-}, \tau_{i}\right) \\
& \quad=\Phi\left(\tau_{i}^{+}\right)(I+K) \Phi^{-1}\left(\tau_{i}\right)-\Phi\left(\tau_{i}^{-}\right) K \Phi^{-1}\left(\tau_{i}\right) \\
& \quad=B_{i}+\left(B_{i}-I\right) G\left(\tau_{i}^{-}, \tau_{i}\right) .
\end{aligned}
$$

To see (b), we write for $s \neq \tau_{i}$,

$$
\begin{aligned}
G\left(s^{+}, s\right)-G\left(s^{-}, s\right)= & \Phi\left(s^{+}\right)(I+K) \Phi^{-1}(s) \\
& -\Phi\left(s^{-}\right) K \Phi^{-1}(s)=I .
\end{aligned}
$$

For (c), let $t \neq \tau_{i}$; then

$$
\begin{gathered}
\frac{\partial G(t, s)}{\partial t}=\left\{\begin{array}{cc}
\Phi^{\prime}(t)(I+K) \Phi^{-1}(s) \\
=J H(t) \Phi(t)(I+K) \Phi^{-1}(s), & s<t \\
\Phi^{\prime}(t) K \Phi^{-1}(s) & s \geq t \\
=J H(t) \Phi(t) K \Phi^{-1}(s), &
\end{array}\right. \\
\begin{aligned}
\frac{\partial G\left(s^{+}, s\right)}{\partial t}-\frac{\partial G\left(s^{-}, s\right)}{\partial t} & =J H(s) \Phi(s)(I+K) \Phi^{-1}(s) \\
& -J H(s) \Phi(s) K \Phi^{-1}(s) \\
& J H(s) .
\end{aligned}
\end{gathered}
$$

Next, we consider (G4). By definition, it is easy to see that $G(t, s)$ is left continuous function at $t=\tau_{i}$. Let us consider the interval $\left[t_{1}, s\right)$. The later is similar. The first equation in (39) is direct consequences of (c) and the definition of $G(t, s)$. Clearly,

$$
\begin{aligned}
& G\left(\tau_{i}^{+}, s\right)=\Phi\left(\tau_{i}^{+}\right) K \Phi^{-1}(s) \\
& \quad=B_{i} \Phi\left(\tau_{i}^{-}\right) K \Phi^{-1}(s)=B_{i} G\left(\tau_{i}^{-}, s\right), \\
& M G\left(t_{1}, s\right)+N G\left(t_{2}, s\right) \\
& =M \Phi\left(t_{1}\right) K \Phi^{-1}(s) \\
& \quad+N \Phi\left(t_{2}\right)(I+K) \Phi^{-1}(s) \\
& =\left[M \Phi\left(t_{1}\right)+N \Phi\left(t_{2}\right)\right] K \Phi^{-1}(s) \\
& \quad+N \Phi\left(t_{2}\right) \Phi^{-1}(s)=0 .
\end{aligned}
$$

The proofs of (G5) and (G6) are similar to (a) and (G4), respectively.

Remark 3. One can easily rewrite Green's function (pair) in terms of the solutions of system (8a), (8b). Indeed,

$$
\begin{aligned}
K= & -\left[M \Phi\left(t_{1}\right)+N \Phi\left(t_{2}\right)\right]^{-1} N \Phi\left(t_{2}\right) \\
= & \frac{1}{x_{1}\left(t_{1}\right) x_{2}\left(t_{2}\right)-x_{1}\left(t_{2}\right) x_{2}\left(t_{1}\right)} \\
& \times\left[\begin{array}{cc}
x_{1}\left(t_{2}\right) x_{2}\left(t_{1}\right) & x_{2}\left(t_{1}\right) x_{2}\left(t_{2}\right) \\
-x_{1}\left(t_{1}\right) x_{1}\left(t_{2}\right) & -x_{1}\left(t_{1}\right) x_{2}\left(t_{2}\right)
\end{array}\right],
\end{aligned}
$$


and since

$$
\begin{aligned}
\operatorname{det} \Phi(t)= & \operatorname{det} \Phi(0) \exp \left(\int_{0}^{t} \operatorname{trace}(J H(s)) \mathrm{d} s\right) \\
& \times \prod_{i=1}^{p} \operatorname{det} B_{i}=\prod_{i=1}^{p} \alpha_{i}^{2},
\end{aligned}
$$

we may write

$$
\begin{aligned}
\Phi^{-1}(t) & =\frac{1}{\operatorname{det} \Phi(t)}\left[\begin{array}{cc}
u_{2}(t) & -x_{2}(t) \\
-u_{1}(t) & x_{1}(t)
\end{array}\right] \\
& =\left(\prod_{i=1}^{p} \alpha_{i}^{-2}\right)\left[\begin{array}{cc}
u_{2}(t) & -x_{2}(t) \\
-u_{1}(t) & x_{1}(t)
\end{array}\right] .
\end{aligned}
$$

\section{The Main Result}

Our main result is the following theorem.

Theorem 4. Let (i)-(iii) hold. If

$$
\begin{aligned}
& \exp \left(\int_{t_{1}}^{t_{2}}|a(t)| d t\right)\left[\int_{t_{1}}^{t_{2}} b(t) d t\right] \\
& \quad \times\left[\int_{t_{1}}^{t_{2}} c^{+}(t) d t+\sum_{t_{1} \leq \tau_{i}<t_{2}}\left(\frac{\beta_{i}}{\alpha_{i}}\right)^{+}\right] \\
& <4
\end{aligned}
$$

then BVP (6a), (6b), and (6c) has a unique solution $(x(t), u(t))$. Moreover, $y=(x(t), u(t))$ is expressible as

$$
y(t)=w(t)+\int_{t_{1}}^{t_{2}} G(t, s) f(s) d s+\sum_{t_{1} \leq \tau_{i}<t_{2}} \widetilde{G}\left(t, \tau_{i}^{+}\right) a_{i},
$$

where

$$
w(t)=\Phi(t)\left[M \Phi\left(t_{1}\right)+N \Phi\left(t_{2}\right)\right]^{-1} \eta, \quad \eta=(A, B)^{T},
$$

and Green's function pair $(G, \widetilde{G})$ is given by (38).

Proof. We first prove the uniqueness. It suffices to show that the homogeneous BVP $(8 \mathrm{a})-(8 \mathrm{c})$ has only the trivial solution. Let $x(t) \not \equiv 0$ on $\left(t_{1}, t_{2}\right)$. By Theorem 1 , we see that Lyapunov type inequality (11) holds contradicting the inequality (47). Thus $x(t)=0$ for all $t \in\left[t_{1}, t_{2}\right]$. Moreover, by (6a), (6b), and (6c) we have

$$
b(t) u=0, \quad t \neq \tau_{i},
$$

which results in $u(t)=0$ for $t \neq \tau_{i}$. Taking limit we see that $u\left(\tau_{i}^{ \pm}\right)=0$. As a result we obtain $(x(t), u(t))=(0,0)$ for all $t \in\left[t_{1}, t_{2}\right]$. This completes the uniqueness of the solutions.
For the existence, we start with the variation of parameters formula and write the general solution of system (6a), $(6 b)$ as

$$
\begin{aligned}
y(t)= & \Phi(t) c+\int_{t_{1}}^{t} \Phi(t) \Phi^{-1}(s) f(s) \mathrm{d} s \\
& +\sum_{t_{1} \leq \tau_{i}<t} \Phi(t) \Phi^{-1}\left(\tau_{i}^{+}\right) a_{i} .
\end{aligned}
$$

Clearly, the boundary condition is satisfied if

$$
\begin{aligned}
& {\left[M \Phi\left(t_{1}\right)+N \Phi\left(t_{2}\right)\right] c} \\
& =\eta-N \Phi\left(t_{2}\right) \\
& \quad \times\left[\int_{t_{1}}^{t_{2}} \Phi^{-1}(s) f(s) \mathrm{d} s+\sum_{t_{1} \leq \tau_{i}<t_{2}} \Phi^{-1}\left(\tau_{i}^{+}\right) a_{i}\right],
\end{aligned}
$$

where $\eta=(A, B)^{T}$.

Since we have the uniqueness of solutions, the matrix $M \Phi\left(t_{1}\right)+N \Phi\left(t_{2}\right)$ must have an inverse. Setting

$$
K=-\left[M \Phi\left(t_{1}\right)+N \Phi\left(t_{2}\right)\right]^{-1} N \Phi\left(t_{2}\right),
$$

we may solve $c$ from (52) uniquely:

$$
\begin{aligned}
c= & {\left[M \Phi\left(t_{1}\right)+N \Phi\left(t_{2}\right)\right]^{-1} \eta } \\
& +K\left[\int_{t_{1}}^{t_{2}} \Phi^{-1}(s) f(s) \mathrm{d} s+\sum_{t_{1} \leq \tau_{i}<t_{2}} \Phi^{-1}\left(\tau_{i}^{+}\right) a_{i}\right] .
\end{aligned}
$$

Hence,

$$
\begin{aligned}
y(t)= & \Phi(t)\left[M \Phi\left(t_{1}\right)+N \Phi\left(t_{2}\right)\right]^{-1} \eta+\Phi(t)(I+K) \\
& \times\left[\int_{t_{1}}^{t} \Phi^{-1}(s) f(s) \mathrm{d} s+\sum_{t_{1} \leq \tau_{i}<t} \Phi^{-1}\left(\tau_{i}^{+}\right) a_{i}\right] \\
& +\Phi(t) K\left[\int_{t}^{t_{2}} \Phi^{-1}(s) f(s) \mathrm{d} s+\sum_{t \leq \tau_{i}<t_{2}} \Phi^{-1}\left(\tau_{i}^{+}\right) a_{i}\right] .
\end{aligned}
$$

Therefore the unique solution of the BVP (6a)-(6c) can be expressed as

$$
\begin{aligned}
y(t)= & w(t)+\int_{t_{1}}^{t_{2}} G(t, s) f(s) \mathrm{d} s \\
& +\sum_{t_{1} \leq \tau_{i}<t_{2}} \widetilde{G}\left(t, \tau_{i}^{+}\right) a_{i} .
\end{aligned}
$$


Let us now consider the BVP (10a), (10b), (10c), and (10d). In this case it is not difficult to see that the corresponding Green's function (pair) becomes

$$
\begin{gathered}
G(t, s)= \begin{cases}\psi(t)(I+K) \Psi^{-1}(s) \frac{1}{p(s)} e_{2}, & s<t, \\
\psi(t) K \Psi^{-1}(s) \frac{1}{p(s)} e_{2}, & s \geq t,\end{cases} \\
\widetilde{G}\left(t, \tau_{i}^{+}\right)= \begin{cases}\psi(t)(I+K) \Psi^{-1}\left(\tau_{i}^{+}\right), & \tau_{i}<t, \\
\psi(t) K \Psi^{-1}\left(\tau_{i}^{+}\right), & \tau_{i} \geq t,\end{cases}
\end{gathered}
$$

where $\psi(t)=\left[\psi_{1}, \psi_{2}\right]$ is the first row of the (Wronskian) matrix:

$$
\begin{gathered}
\Psi(t)=\left[\begin{array}{ll}
\psi_{1}(t) & \psi_{2}(t) \\
\psi_{1}^{\prime}(t) & \psi_{2}^{\prime}(t)
\end{array}\right], \\
K=-\left[M \Psi\left(t_{1}\right)+N \Psi\left(t_{2}\right)\right]^{-1} N \Psi\left(t_{2}\right), \\
e_{2}=[0,1]^{T} .
\end{gathered}
$$

Corollary 5. Suppose that $p$ and $c$ are piece-wise continuous on $\left[t_{1}, t_{2}\right], p(t)>0$, and $\alpha_{i} \neq 0$ for $i=1,2, \ldots, p$. If

$$
\left[\int_{t_{1}}^{t_{2}} \frac{1}{p(t)} d t\right]\left[\int_{t_{1}}^{t_{2}} c^{+}(t) d t+\sum_{t_{1} \leq \tau_{i}<t_{2}}\left(\frac{\beta_{i}}{\alpha_{i}}\right)^{+}\right]<4
$$

then the BVP (10a), (10b), (10c), and (10d) has a unique solution $x(t)$ which is expressible as

$$
x(t)=w(t)+\int_{t_{1}}^{t_{2}} G(t, s) f(s) d s+\sum_{t_{1} \leq \tau_{i}<t_{2}} \widetilde{G}\left(t, \tau_{i}^{+}\right) a_{i},
$$

where

$$
w(t)=\psi(t)\left[M \Psi\left(t_{1}\right)+N \Psi\left(t_{2}\right)\right]^{-1} \eta
$$

and Green's function pair $(G, \widetilde{G})$ is given by (57).

Remark 6. The results in this work are new even if the impulses are absent. The statements of the corresponding theorems are left to the reader.

\section{References}

[1] J. J. Nieto, "Basic theory for nonresonance impulsive periodic problems of first order," Journal of Mathematical Analysis and Applications, vol. 205, no. 2, pp. 423-433, 1997.

[2] J. Li, J. J. Nieto, and J. Shen, "Impulsive periodic boundary value problems of first-order differential equations," Journal of Mathematical Analysis and Applications, vol. 325, no. 1, pp. 226236, 2007.

[3] J. J. Nieto, "Periodic boundary value problems for first-order impulsive ordinary differential equations," Nonlinear Analysis: Theory, Methods \& Applications A, vol. 51, no. 7, pp. 1223-1232, 2002.
[4] J. J. Nieto, "Impulsive resonance periodic problems of first order," Applied Mathematics Letters, vol. 15, no. 4, pp. 489-493, 2002.

[5] A. Cabada, J. J. Nieto, D. Franco, and S. I. Trofimchuk, "A generalization of the monotone method for second order periodic boundary value problem with impulses at fixed points," Dynamics of Continuous, Discrete and Impulsive Systems, vol. 7, no. 1, pp. 145-158, 2000.

[6] J. Li and J. Shen, "Periodic boundary value problems for second order differential equations with impulses," Nonlinear Studies, vol. 12, no. 4, pp. 391-400, 2005.

[7] J. J. Nieto and D. O’Regan, "Variational approach to impulsive differential equations," Nonlinear Analysis: Real World Applications, vol. 10, no. 2, pp. 680-690, 2009.

[8] J. J. Nieto, "Variational formulation of a damped Dirichlet impulsive problem," Applied Mathematics Letters, vol. 23, no. 8, pp. 940-942, 2010.

[9] J. Xiao and J. J. Nieto, "Variational approach to some damped Dirichlet nonlinear impulsive differential equations," Journal of the Franklin Institute, vol. 348, no. 2, pp. 369-377, 2011.

[10] M. Galewski, "On variational impulsive boundary value problems," Central European Journal of Mathematics, vol. 10, no. 6, pp. 1969-1980, 2012.

[11] P. W. Eloe and J. Henderson, "A boundary value problem for a system of ordinary differential equations with impulse effects," The Rocky Mountain Journal of Mathematics, vol. 27, no. 3, pp. 785-799, 1997.

[12] Ö. Uğur and M. U. Akhmet, "Boundary value problems for higher order linear impulsive differential equations," Journal of Mathematical Analysis and Applications, vol. 319, no. 1, pp. 139156, 2006.

[13] A. M. Samoilenko and N. A. Perestyuk, Impulsive Differential Equations, World Scientific, Singapore, 1995.

[14] A. Liapounoff, "Problème général de la stabilité du mouvement," Annales de la Faculté des Sciences de Toulouse pour les Sciences Mathématiques et les Sciences Physiques. Série 2, vol. 9, pp. 203474, 1907, Annals of Mathematics Studies, vol. 17, pp. 203-474, 1947.

[15] G. Sh. Guseinov and B. Kaymakçalan, "Lyapunov inequalities for discrete linear Hamiltonian systems," Computers \& Mathematics with Applications, vol. 45, no. 6-9, pp. 1399-1416, 2003.

[16] X. Wang, "Stability criteria for linear periodic Hamiltonian systems," Journal of Mathematical Analysis and Applications, vol. 367, no. 1, pp. 329-336, 2010.

[17] X.-H. Tang and M. Zhang, "Lyapunov inequalities and stability for linear Hamiltonian systems," Journal of Differential Equations, vol. 252, no. 1, pp. 358-381, 2012.

[18] G. Sh. Guseinov and A. Zafer, "Stability criterion for second order linear impulsive differential equations with periodic coefficients," Mathematische Nachrichten, vol. 281, no. 9, pp. 1273-1282, 2008.

[19] G. Sh. Guseinov and A. Zafer, "Stability criteria for linear periodic impulsive Hamiltonian systems," Journal of Mathematical Analysis and Applications, vol. 335, no. 2, pp. 1195-1206, 2007.

[20] Z. Kayar and A. Zafer, "Stability criteria for linear Hamiltonian systems under impulsive perturbations," http://arxiv.org/abs/ 1101.3094 . 


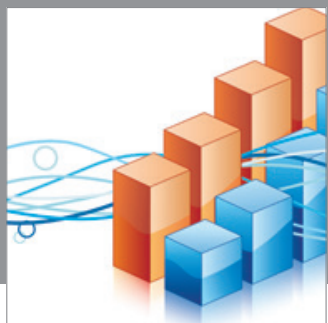

Advances in

Operations Research

mansans

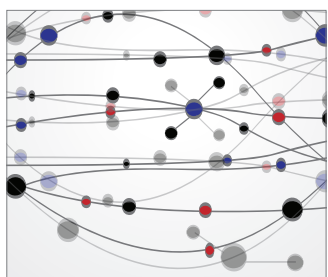

The Scientific World Journal
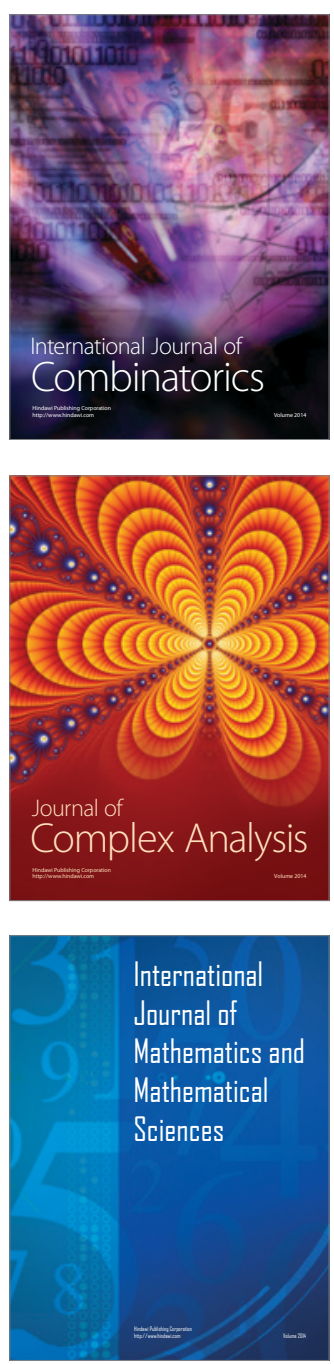
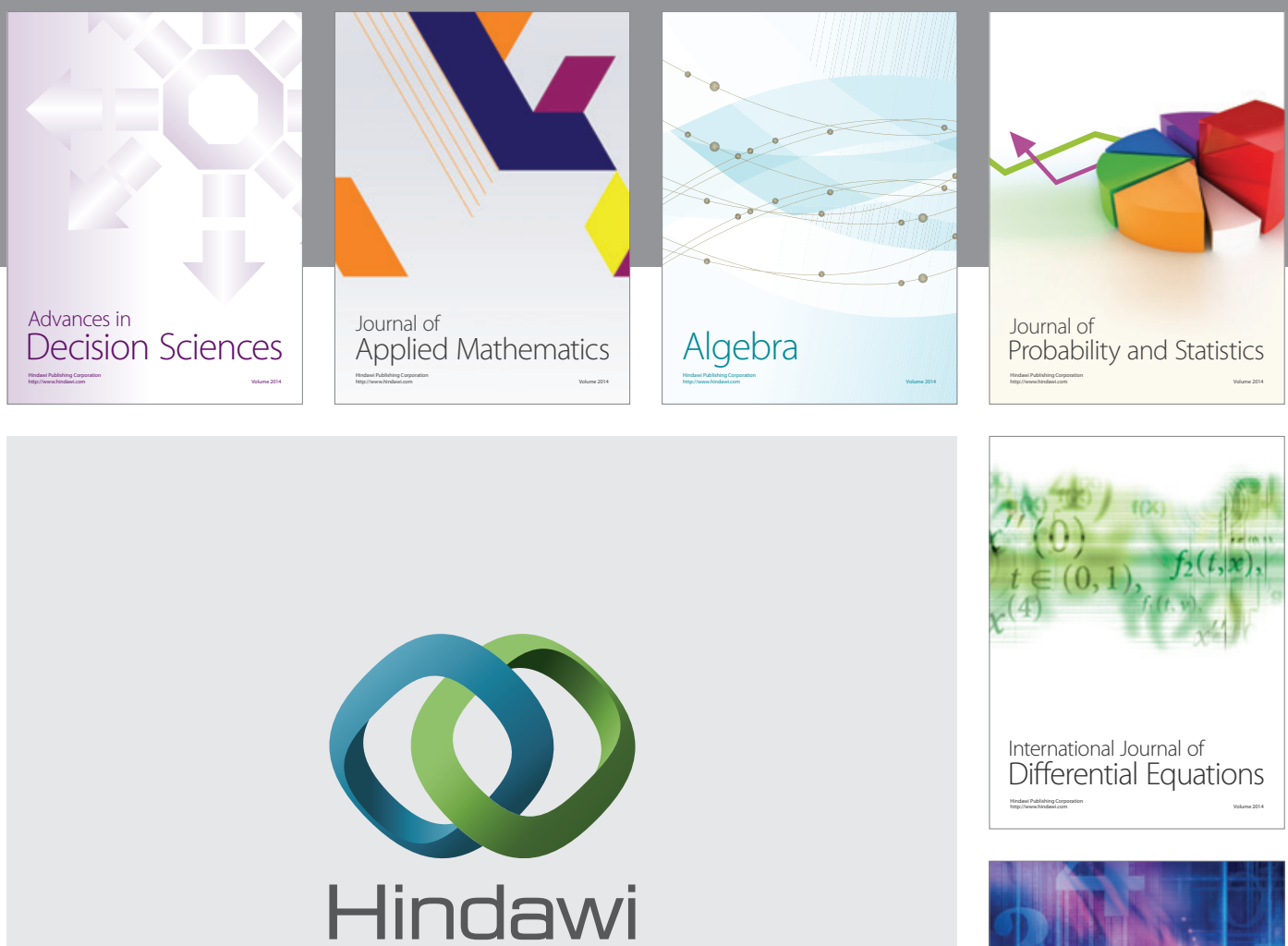

Submit your manuscripts at http://www.hindawi.com
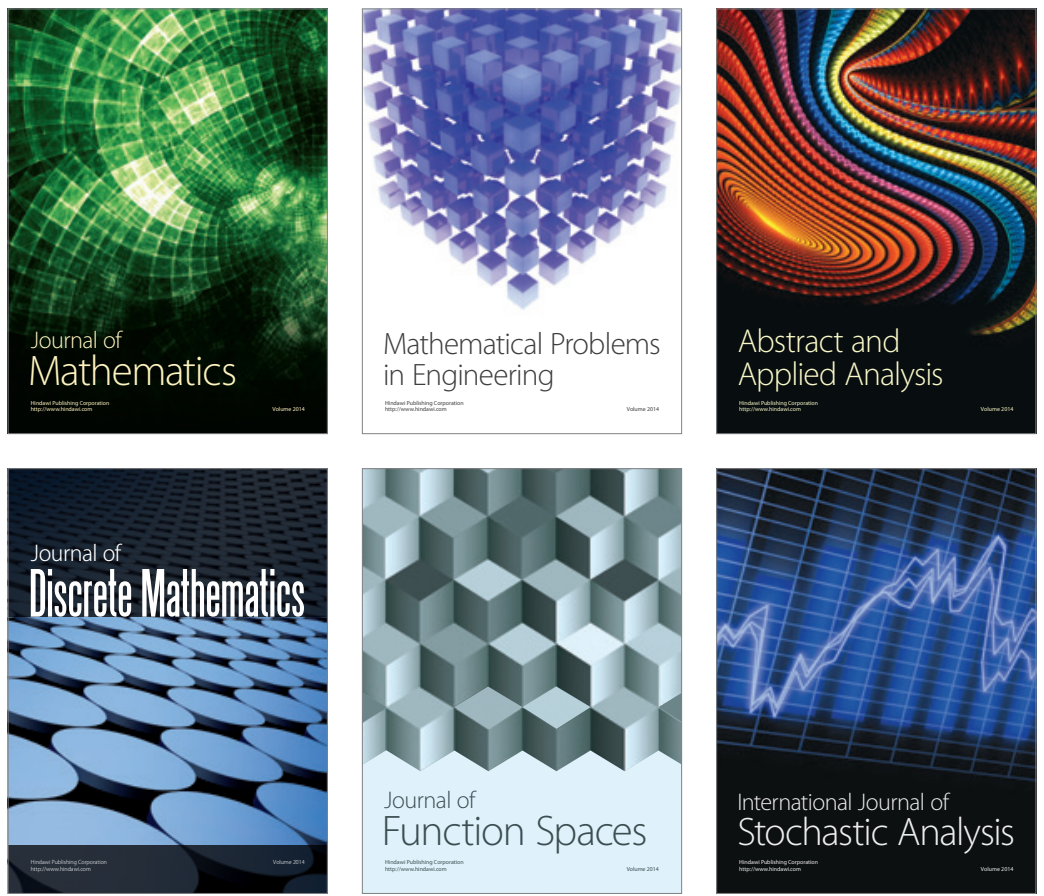

Journal of

Function Spaces

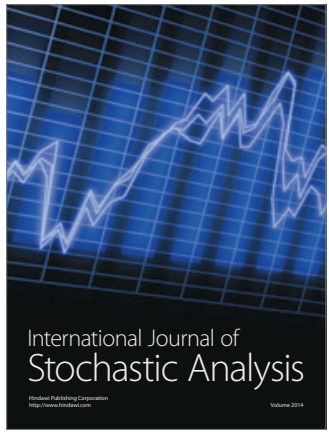

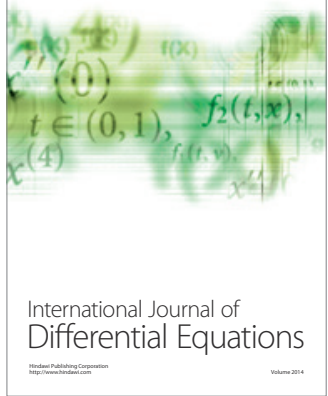
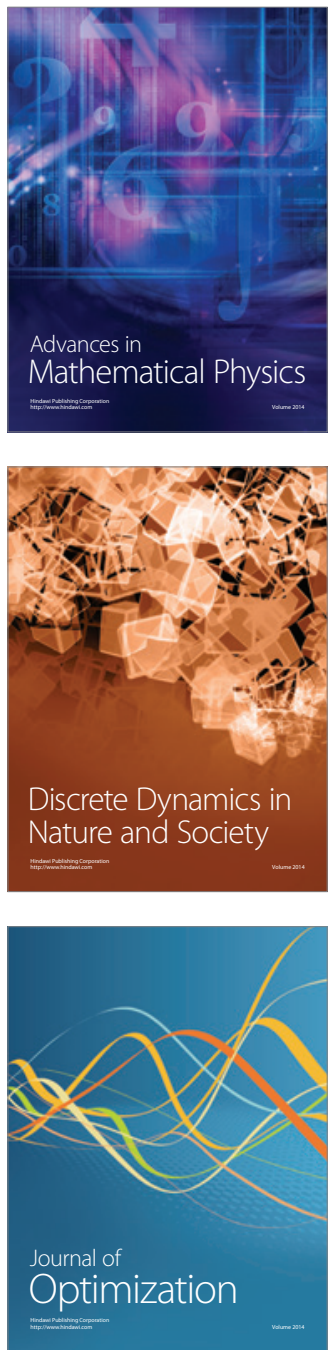